\title{
INTERNET USE FOR CLINICAL DECISION MAKING: MODERATING EFFECTS OF UNCERTAINTY AND EQUIVOCALITY
}

\author{
Paul J. Ambrose, University of Wisconsin-Whitewater, ambrosep@uww.edu \\ Grace J. Johnson, University of Wisconsin-Milwaukee,gracej@uwm.edu
}

\begin{abstract}
The success of clinical decision-making depends on providing the right information at the right time to key clinicians such as physicians. The Internet has tremendously enabled medical information provision for clinical decision-making. However, key factors that influence the use of the Internet for medical information provisioning are not widely known. This research synthesizes the clinical and general decision-making, and information processing literature streams to build a research model and empirically validate the moderating role of uncertainty and equivocality in the use of the Internet for provisioning information for clinical decision-making.
\end{abstract}

Keywords: Internet Use, Clinical Decision-making, Information Processing Theory, Decision Performance

\section{INTRODUCTION}

The commercialization of the Internet in the1990s and the development of the World Wide Web (WWW) architecture paved the way for effective and efficient use of information to support work processes, particularly knowledge intensive work processes. Rapid advances in information and communications technologies since the 1990s not only have increased the easy availability of actionable information, but have also provided a plethora of tools and techniques to analyze and draw deep insights from such information. Today, SMAC (Social, Mobile, Analytics, and Cloud) technologies have harnessed the power of the Internet to move large amounts of information in varied formats, from simple texts to complex audio-visual information, directly into the hands of decision makers with consequent positive impacts in decision-making quality [1].

Clinical Decision Making (CDM) is a knowledge intensive work process that can tremendously benefit from the value adding information provisioning made possible by the Internet and allied SMAC technologies. CDM involves decision-making on diagnosis and prognosis of ailments, and on choice of treatment [18]. Uncertainty (due to lack of information) and equivocality (due to ambiguous information) surround clinical decision-making on account of the probabilistic nature of the relationship among symptoms (patients' presentation of complaints), signs (physicians' interpretations of symptoms) and diseases [7]. Clinical decision-making is a knowledge intensive work process that requires physicians' knowledge to reduce uncertainty and equivocality in the decision-making process so that they arrive at the most appropriate medical decision.

Physicians possess and use an enormous amount of knowledge necessary for clinical decision-making. Physicians' knowledge is accumulated through training, experience, and by reading scientific research. However, the knowledge possessed by physicians tends to get outdated quickly. Medical research generates new information at a rate far greater than the rate at which it can be effectively disseminated to physicians [5, 16]. Physicians find it difficult to access and keep up with current medical research findings, as the volume of information that gets published every year is extensive. As a result, clinical decisions tend to differ among physicians [18].

While Internet enabled information provisioning has the potential to enhance CDM, traditional information processing constraints such as information overload, bounded rationality, uncertainty, and equivocality continue to exist $[4,17]$. Thus, it is imperative to empirically investigate if there are any positive impacts of Internet enabled information provisioning on the clinical decision-making process, and if there are, how those benefits materialize. In this research we undertake an empirical study to examine if Internet use by physicians for information provisioning positively impacts the quality and timeliness of clinical decision-making. Specifically, we address the following research questions:

1. What is the appropriate theoretical perspective to examine the impact of Internet use in CDM? Through a literature review, we identify salient elements from information processing and clinical decisionmaking theories that help us understand the impact of Internet use in CDM. 
2. What salient factors influence the impact of Internet use in CDM?

Based on our literature review, we develop a moderating model to show the role played by uncertainty and equivocality in defining the relationship between Internet use and CDM quality and timeliness

3. Is there empirical validity to the nature of the relationship between Internet use and CDM quality and timeliness?

We undertake a survey of physicians from across the United States to establish empirical evidence for the moderating role of uncertainty and equivocality.

The rest of the paper proceeds as follows. The next section presents our theoretical arguments for our research premise. The section following presents the empirical study and its results. The paper concludes with a discussion, and directions for future research.

\section{THEORETICAL BACKGROUND}

We examine the clinical decision-making, information processing, and decision-making literature streams to examine the nature of the relationship between Internet use and clinical decision-making outcomes, namely quality and timeliness of the decision. Information processing theory is the meta theory guiding this research.

\section{Clinical Decision-Making}

As noted earlier, CDM involves three distinct activities - diagnosis, prognosis, and choice of treatment. Diagnosis involves the identification of the nature and causes of the primary and associated ailments. Prognosis is the judgment on the progress of the ailment, while the choice of treatment refers to the decision made on the best possible treatment for the ailment. Professional clinical decision-makers such as physicians, and registered nurse practitioners make clinical decisions.

While factors like physician and characteristics, patient factors, practice settings, environmental constraints, and communication channels can influence clinical decision-making, information remains a critical influencer in clinical decision-making [18]. Models of clinical decision-making have focused on the role of information in clinical decision-making and Eddy's basic model [6] shown in Figure 1 below highlights how information is vital to the CDM.

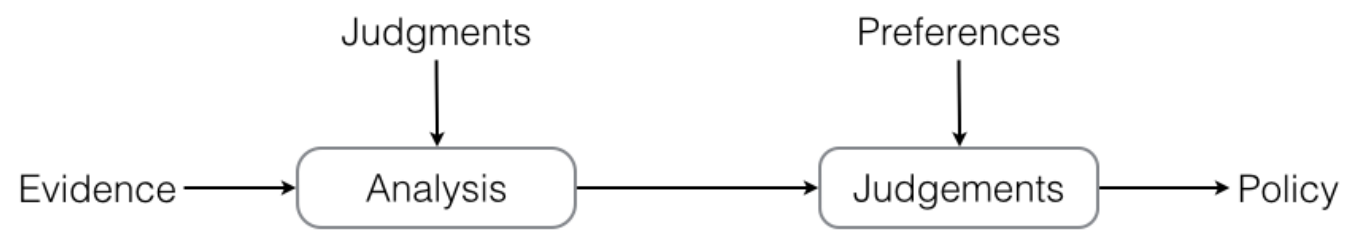

Figure 1: Basic Clinical Decision-Making Model

Figure 1 depicts evidence gathering as stage 1, where the clinical decision-maker collects and analyses available evidence on the benefits, risks and costs of each clinical outcome. A certain degree of subjective judgment characterizes this stage given the incomplete nature of medical evidence. The output of step 1 forms the input for step 2, and here clinicians and patients make judgments on the suitability of each outcome in the first stage. Uncertainty and equivocality exists in CDM and their levels vary based on contextual factors such as the nature of the ailment. Eddy proposed that the quality of the final choice or decision could be improved by increasing the amount and the quality of evidence, which highlights how information is critical in clinical decision-making.

The shelf life of knowledge acquired in medical school is estimated to be two years and it is also estimated that the medical knowledge base doubles every 19 years [19]. There are about 360,000 medical journal publications every year and that is almost 1,000 new articles added to medical literature each day. A fourfold increase in medical knowledge during a physician's lifetime necessitates clinicians to seek significant information to support their clinical decision-making. 


\section{Information Processing Theory}

Our research uses the information processing approach as the organizing framework, which at a broad level, deals with activities associated with collecting, storing, manipulating and retrieving information so that it can be used effectively in a user defined context. Information processing helps provide appropriate context-focused information to accomplish tasks. This approach has been used as a meta-theory to explain various information related aspects in a variety of disciplines like cognitive psychology, economics, marketing, organization studies and information systems.

A key focus of the information processing approach is to examine the role of uncertainty and equivocality in decision-making contexts that impacts the level of information processing needs. When a task, especially a knowledge-intensive task is shrouded in uncertainty, the need for information processing increases. Uncertainty refers to lack or absence of information. As information relevant to the context increases, uncertainty decreases. However, there are situations where an increase in information does not translate into effective task performance as more information can lead to information overload or when the information that is being provided for a particular task is ambiguous or equivocal. Equivocality leads to multiple and conflicting interpretations of a task [4]. Daft and Lengel [4] suggested that rich information, as one that is able "to change understanding within a time interval", can help reduce equivocality.

\section{Decision-making Performance}

Decision making performance is a multidimensional construct [13]. The information systems literature indicates that decision quality, decision timeliness, decision process satisfaction, and decision outcome satisfaction are commonly used composite measures of decision-making performance $[11,13,14,18]$. Performance can be evaluated either objectively or subjectively $[12,13]$. Objective measures of performance are typically quality and timeliness, where actual values of these measures are compared with desired values. However quality and timeliness can become subjective measures when these are measured as perceived quality and timeliness from the point of view of decisionprocess participants or stakeholders. On the other hand, decision satisfaction is a perceived measure of decisionmaking performance.

Objective measures are generally preferred, as subjective measures tend to have the decision-maker or decision stakeholder bias built into the measure. While it is desirable to have objective measures of decision-making performance, it might not be possible to obtain these in all instances. If that be the case, then subjective measures will have to be used to evaluate the performance of decision-making. If designed and measured properly, subjective measures can be a good surrogate for objective measures of decision-making performance [12].

\section{RESEARCH MODEL}

The research model built and empirically validated in this research is shown in Figure 2 below. The model is premised from the theoretical background summarized earlier that CDM needs the provisioning of information to enhance clinical decision making performance. While multiple factors can impact CDM performance, our research model focuses on the role of information. Specifically, we focus on how information provisioning is moderated by the levels of uncertainty and equivocality in the CDM context. We are interested in understanding the factors that affect the direction and/or strength of the relation between Internet use for information provision in CDM contexts and CDM performance, and as such, uncertainty and equivocality are modeled as moderators and not as mediators. Mediating relationships are used to explain the relationship between two constructs or how/why such effects occur, while moderating relationships specify when certain effects hold by examining the strength and direction of the relationship.

As noted earlier, CDM performance is a multidimensional construct that can be measured either objectively or subjectively, and from a scope and parsimony perspective we include subjective measures of decision quality and decision timeliness as measures of CDM performance. Performance of clinical decision-making is defined as the extent to which the decision achieves the objectives established by the physician and patient. Uncertainty and equivocality in clinical decision-making is defined as the extent to which the information available for clinical 
decision-making is able to generate unambiguous solution(s) to the problem. Internet Use for Clinical Decision Making is defined as the extent to which information is sought using the Internet by clinicians for CDM.

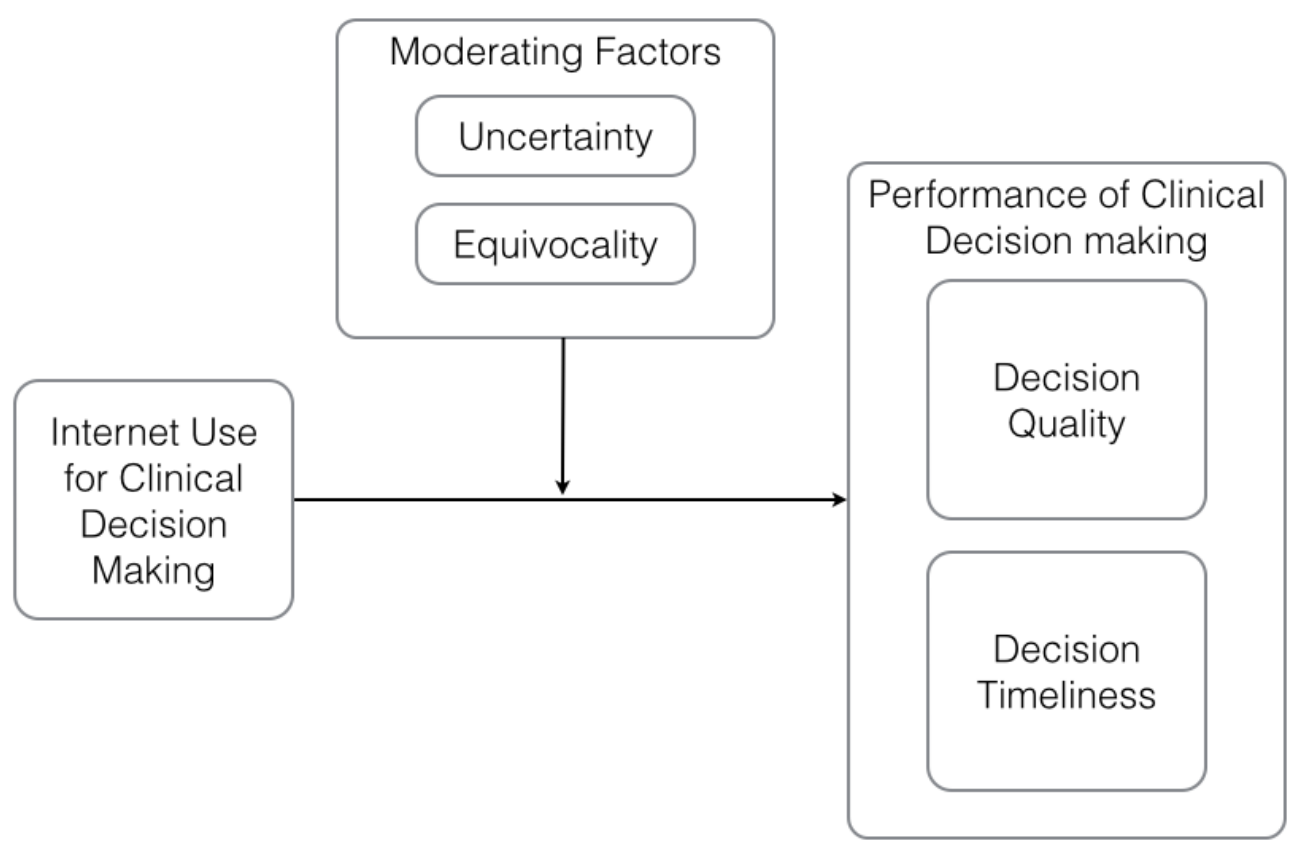

Figure 2: Research Model

\section{Research Design and Data Collection}

\section{EMPIRICAL VALIDATION}

Survey instrument design and model validation was undertaken using a two-stage empirical study. The first stage involved interviewing 41 physicians in both private and academic practice to generate construct items and establish their face validity, as there were no prior established indicators for these constructs in the clinical decision-making context. In the second stage, the research model was empirically validated through a self-administered mail-in survey. Closed-ended questions were developed for each construct (summarized in Table 1). Responses to construct items were elicited on a 5-point Likert type scale anchored at "Never" and "Very Frequently" at each end. The development and validation of the instrument was done along the lines suggested by Churchill, and Sethi and King $[3,15]$ and included pilot testing.

To elaborate, interviews indicated that physicians associated 'detailed', 'scientific', 'abstracted', 'lay', 'current', and 'archived' to denote the various types of medical information they obtained on the Internet. Accordingly, the Internet Use of Clinical Decision-Making construct was developed as a reflective construct with the items listed in Table 1. Uncertainty is the lack of information for a task, and equivocality, the presence of variety of information, leading to conflicting results. Given the probabilistic nature of the relationship among symptoms, signs, and diseases [7] we conceptualized uncertainty being high when patients don't present their symptoms fully, when signs, treatment protocols, or prognosis are incomplete, or when the physician is unable to assemble all evidence required for clinical decision-making. Similarly, equivocality is high when a set of signs point to many diseases, when multiple treatment options are available, or when a treatment has multiple outcomes. Equivocality is thus high when the clinical evidence can be interpreted in many ways.

Decision quality is the extent to which the decision taken conforms to the actual decision to be taken by maximizing benefits and minimizing risks within cost constraints. Physicians, patients, and insurance companies are the key stakeholders that are concerned with the decision. Fulfillment of these stakeholder objectives will indicate whether the actual decision was made from the point of view of these stakeholders. Timeliness refers to the speed of clinical decisions taken and has two facets. A physician could make a timely decision if the decision was made within the normally accepted time frame for that decision. However, within a time frame, some physicians could make faster 
decisions than others. Thus, there would be an appropriate time taken to make decisions. Using these two facets, the timeliness items were constructed for the three stakeholders of clinical decision-making.

997 physicians in the US Midwest in private and academic practice, in both rural and urban settings were identified as potential survey respondents. A mailing of the survey instrument with a reminder in two weeks resulted in 248 useful responses, which amounts to approximately a $25 \%$ response rate. Preliminary data screening [8] did not reveal any issues relating to coding errors, missing values, implausible values, data nonnormality, and nonresponse bias. The multi-item scales were also assessed for unidimensionality, reliability, and convergent and discriminant validities [8] with no discrepancies.

\section{Data Analysis and Results}

Table 1 summarize the preliminary data analysis before the structural model was evaluated using the Partial Least Square (PLS), a variance based Structural Equation Modeling (SEM) technique [9]. PLS can simultaneously evaluate both the measurement and the structural model. Measurement model evaluation did not reveal any discrepancies and the results were in line with the preliminary data analysis.

Table 1: Preliminary Analysis: Unidimensionality and Reliability

\begin{tabular}{|c|c|c|c|}
\hline Items & $\begin{array}{c}\text { Factor } \\
\text { Loading }\end{array}$ & $\begin{array}{l}\text { Variance } \\
\text { Explained }\end{array}$ & $\begin{array}{c}\text { Cronbach's } \\
\alpha\end{array}$ \\
\hline \multicolumn{4}{|c|}{ Internet Use for Clinical Decision-Making } \\
\hline 1. I obtain current medical information on the Internet. & 0.934 & \multirow[t]{6}{*}{$74.3 \%$} & \multirow[t]{6}{*}{0.93} \\
\hline 2. I obtain archived medical information on the Internet. & 0.905 & & \\
\hline 3. I obtain detailed medical information on the Internet. & 0.882 & & \\
\hline 4. I obtain abstracted medical information on the Internet. & 0.922 & & \\
\hline 5. I obtain scientific medical information on the Internet. & 0.927 & & \\
\hline 6. I obtain lay medical information on the Internet. & 0.528 & & \\
\hline \multicolumn{4}{|l|}{ Uncertainty (* reverse coded) } \\
\hline 1. *My patients present their symptoms completely. & 0.567 & \multirow[t]{6}{*}{$53.2 \%$} & \multirow[t]{6}{*}{0.81} \\
\hline 2. *The signs of a disease are well defined in my practice. & 0.818 & & \\
\hline 3. *Treatment protocols are well defined in my practice. & 0.830 & & \\
\hline 4. *The prognosis, given a treatment, is well defined in my practice. & 0.844 & & \\
\hline 5. *I can relate my patients' symptoms to signs of diseases. & 0.723 & & \\
\hline 6. * I can collect all the evidence I need to make a clinical decision. & 0.530 & & \\
\hline \multicolumn{4}{|l|}{ Equivocality } \\
\hline 1. I can relate a set of signs exhibited by a patient to many diseases. & 0.662 & \multirow[t]{4}{*}{$57.5 \%$} & \multirow[t]{4}{*}{0.75} \\
\hline 2. I can treat a patient's disease in many ways. & 0.827 & & \\
\hline 3. A treatment I choose for a patient may have many outcomes. & 0.793 & & \\
\hline 4. I can interpret the evidence from patients in more than one way. & 0.742 & & \\
\hline \multicolumn{4}{|l|}{ Decision Quality } \\
\hline 1. My clinical decisions help achieve my personal objectives. & 0.854 & \multirow[t]{3}{*}{$52.6 \%$} & \multirow[t]{3}{*}{0.55} \\
\hline 2. My clinical decisions help achieve my patients' objectives. & 0.509 & & \\
\hline $\begin{array}{l}\text { 3. My clinical decisions help achieve the insurance companies' } \\
\text { objectives. }\end{array}$ & 0.767 & & \\
\hline \multicolumn{4}{|l|}{ Decision Timeliness } \\
\hline 1. I make timely clinical decisions. & 0.769 & \multirow[t]{4}{*}{$34.0 \%$} & \multirow[t]{4}{*}{0.70} \\
\hline 2. My patients make timely health care decisions. & 0.529 & & \\
\hline $\begin{array}{l}\text { 3. I take the appropriate amount of time for making clinical } \\
\text { decisions. }\end{array}$ & 0.847 & & \\
\hline 4. My patients take time for making health care decisions. & 0.607 & & \\
\hline
\end{tabular}


Structural model evaluation results are summarized in Table 2.

Table 2: Structural Model Moderating Effects

\begin{tabular}{|l|l|l|}
\hline Moderating Effects & Decision Quality & Decision Timeliness \\
\hline Internet Use * Uncertainty & Positive & Positive \\
\hline Internet Use * Equivocality & Negative & Negative \\
\hline \multicolumn{2}{|l|}{} \\
\hline Model R ${ }^{2}$ : Quality 22.5\%, Timeliness 45.6\% \\
\hline
\end{tabular}

PLS analysis was done with SmartPLS 2.0 using standardized data. The standard procedure suggested by Chin et al [2] for handling moderating effects in PLS was used to test for moderator significance. The structural model analysis interestingly did not show up direct effects of Internet Use on both Decision Quality and Decision Timeliness as significant effects, even at the 0.05 significance level. However, both moderators had significant effects on both CDM outcome variables $(\mathrm{p}<0.01)$, though the direction of each was different. Interpreting the directional signs, it appears that as uncertainty increased, Internet use also increased. However, as equivocality increased, Internet use decreased. Model $\mathrm{R}^{2}$ on both quality and timeliness are also shown in Table 2.

\section{DISCUSSION AND CONCLUSIONS}

The availability of the right information at the right time is critical for clinical decision-making. Clinical decisionmaking requires the provisioning of information to enhance CDM performance, and the informational needs are exacerbated given the tremendous rate at which medical knowledge increases. This research has empirically established the influence of uncertainty and equivocality as key moderators. High levels of uncertainty increase Internet Use to obtain information for clinical decision-making. However, it appears clinicians are wary of increasing information provisioning for CDM. This is understandable, particularly if more information contributes to ambiguity. In such situations, it is imperative for higher levels of information processing by information providers to supply rich information for decision makers.

Recent years have witnessed the emergence of the Internet as an important medium for information distribution. While much has been said about the ability of the Internet to provide support for decision-making through information provisioning, it is important that this medium be used appropriately to elicit desired results. Our research indicates that in cases of uncertainty, the use of the Internet to obtain medical information can actually help improve decision quality and timeliness. However, when the clinical decision-making context is fraught with ambiguity, the use of the Internet for information provisioning for clinical decision-making can actually be counterproductive, and detrimental to decision quality and timeliness.

This raises key issues for the healthcare community. First, physicians and other health care practitioners should become well cognizant of the capabilities and limitations of the various Internet sources that are available, and the appropriate use of these sources in specific contexts. Healthcare practitioners are require to participate in continuing education programs, which are predominantly focused on keeping abreast with developments in the field. It would be useful to expand such continuing education programs to include the use of new technologies particularly Internet based information sources to enhance clinical decision-making. Second, it also opens new opportunities for providers of such information to work closely with the healthcare community to identify and create information sources that cater to uncertainty and equivocal contexts differently. Particularly in contexts of ambiguity, these providers can offer value added service by designing and implementing technologies that help reduce ambiguity.

Today, medical information is being made widely available to the patient population. Patients, as a result, are becoming 'informed patients' [10]. Further, patients are getting frustrated with physicians who are not communicative. Patients increasingly want to discuss more about their ailments with their physicians based on the information that they gather from the Internet. As a result, physician-patient communication is becoming important. Patients are in a position to cross-check diagnoses or even physician credentials on the Internet. Physician understanding of what patients are looking at on the Internet can help both parties understand each other better and help build physician-patient relationships. All this can lead to increased patient satisfaction, and even retention. Thus 
a patient-oriented physician-patient relationship model is important in this information age. Success depends on how well physicians understand and use Internet information sources to enhance CDM.

Further, physicians like to be sure of content authenticity before they use a portal or recommend it to their patients. Since they are time-pressed, they are unable to validate the content of most sites. In this situation, they tend to trust sites from professional societies more than commercial sites. Conveying content authenticity is the need of the hour for commercial medical information providers. Such providers can add value by understanding the nature of uncertainty and equivocality, and design systems that can provide rich context specific information to help enhance physician decision-making.

While this research helped empirically validate the moderating role of uncertainty and equivocality on Internet use for information provisioning, the research does have limitations that can be addressed in future research. The research design was based on a key informant approach. Data collection from physicians and their patients would have been ideal, especially when data can be obtained from a physician-patient dyad, to fully understand CDM performance. However, operationalizing such a design would be difficult, as a physician-patient dyad needs the cooperation of both physicians and their patients. Quality, and timeliness should ideally be measured objectively instead of measuring these dimensions subjectively through physicians. Also, this empirical study was done in the US Midwest. Collecting data at a national or an international level would help further validate the model. The research model can also be expanded to include other salient CDM factors. However, these limitations do not detract from the main thesis that this research sought to theoretically develop and empirically validate.

\section{REFERENCES}

1. Ambrose, P.J. and G.J. Johnson. (2014). Beyond Gamification: Customer as Players of Serious Games For Innovation. Cutter IT Journal. 27(5): p. 6-12.

2. Chin, W.W., B.L. Marcolin, and P.R. Newsted. (2003). A Partial Least Squares Latent Variable Modeling Approach for Measuring Interaction Effects: Results from a Monte Carlo Simulation Study and an Electronic-Mail Emotion/Adoption Study. Information Systems Research. 14(2): p. 189-217.

3. Churchill, G.A. (1979). A Paradigm for Developing Better Measures of Marketing Constructs. Journal of Marketing Research. 16: p. 64-73.

4. Daft, R.L., R.H. Lengel, and L.K. Trevino. (1987). Message Equivocality, Media Selection, and Manager Performance: Implications for Information Systems. MIS Quarterly. 11(3): p. 355-366.

5. Detmer, W.M. and E.H. Shortliffe. (1997). Using the Internet to improve knowledge diffusion in medicine. Communications of the ACM. 40(8): p. 101-108.

6. Eddy, D.M. (1990). Clinical decision making: from theory to practice. Anatomy of a decision. JAMA. 263(3): p. 441-3.

7. Griffin, T., S. Schwartz, and K. Sofronoff. (1998). Implicit Processes in Medical Diagnosis, in Implicit and Explicit Mental Processes, K. Kirsner, et al., Editors. 1998, Lawrence Erlbaum Associates Publishers: Mahwah, NJ. p. 329-341.

8. Hair, J.F. (2010). Multivariate data analysis. 7th ed., Upper Saddle River, NJ: Prentice Hall. xxviii, 785 p.

9. Hair, J.F., et al. (2014). A primer on partial least squares structural equations modeling (PLS-SEM). Los Angeles: SAGE. xvi, $307 \mathrm{p}$.

10. Johnson, G.J. and A. Ramaprasad. (2000). Patient-Physician Relationships in the Information Age. Marketing Health Services. 20(1): p. 20-27.

11. Mennecke, B.E. and J.S. Valacich. (1998). Information is what you make of it: The influence of group history and computer support on information sharing, decision quality, and member perceptions. Journal of Management Information Systems: JMIS. 15(2): p. 173-197.

12. Reyes, A. and P. Rosso. (2012). Making objective decisions from subjective data: Detecting irony in customer reviews. Decision Support Systems. 53(4): p. 754-760.

13. Ross, J.W., C.M. Beath, and A. Quaadgras. (2013). You May Not Need Big Data After All. Harvard Business Review. 91(12): p. 90-98.

14. Saunders, C. and S. Miranda. (1998). Information acquisition in group decision making. Information \& Management. 34(2): p. 55-74.

15. Sethi, V. and W.R. King. (1991). Construct Measurement in Information Systems Research: An Illustration in Strategic Systems. Decision Sciences. 22(3): p. 455-472. 


\section{Issues in Information Systems}

Volume 15, Issue II, pp. 223-230, 2014

16. Sherwill-Navarro, P.J. and A.L. Wallace. (2004). Research on the value of medical library services: does it make an impact in the health care literature? Journal of the Medical Library Association. 92(1): p. 34-45.

17. Simon, H.A. (1995). The information-processing theory of mind. The American Psychologist. 50(7): p. 507-508.

18. Sox, H.C., M.C. Higgins, and D.K. Owens. (2013). Medical decision making. 2nd ed., Chichester, West Sussex, UK: John Wiley \& Sons. 364 p.

19. Wyatt, J.C. and F. Sullivan. (2005). Keeping up: learning in the workplace. BMJ. 331(7525): p. 1129-1132. 